\title{
Geometric Calibration and Feldkamp Reconstruction for Offset-Detector CBCT
}

\author{
Matt Hemsley, Rolf Clackdoyle, Elsayed Ali and Tong Xu
}

\begin{abstract}
Accurate cone-beam computed tomography (CBCT) reconstruction requires knowing the true geometric parameters of the scanner, obtained from calibrating the system. Positioning the detector panel offset to the source-detector axis is a technique used by linac-mounted CBCTs in the context of radiation therapy in order to image the full width of the patient over the 360-degree scan. However, offsetting the detector panel increases the difficulty of calibration as the resulting projections will be truncated along their width. We present an extended version of an existing phantom-based geometric calibration method that we have adapted for use in offset detector systems. This calibration method extracts the required geometric parameters from measured projection images of a specific calibration phantom we designed for use in the method. The calibration has been implemented in tandem with a variant of the Feldkamp-DavisKress (FDK) reconstruction algorithm which we have modified to both integrate our calibration and reconstruct width-truncated projections. The calibration and modified FDK algorithm are validated by the successful reconstruction of a simulated SheppLogan phantom generated with misaligned detector geometry (detector panel shifting, and variable detector panel rotation along the 360-degree scan). The method also applies to other types of detector misalignment. A physical version of the phantom has been constructed to validate the method with real data in the future.
\end{abstract}

\section{INTRODUCTION}

Accurate cone-beam computed tomography (CBCT) reconstruction is crucially dependent on knowing the true geometric parameters of the scanner (geometric calibration). In the context of linac-mounted CBCTs used for image-guided radiation therapy (IGRT), it is common to position the detector panel offset to the source-detector axis to image the full width of the patient over the 360-degree scan [1] (Fig. 1). However, offsetting the detector increases the difficulty of geometric calibration and reconstruction as the resulting projections will be truncated along their width. For phantombased geometric calibration methods, this truncation implies that the full calibration object will not be visible in any single

Manuscript received December 13, 2019. This work was supported in part by the Natural Sciences and Engineering Research Council (Canada) Discovery Grants RGPIN 342079-2013 and 315071-2017.

M. Hemsley was with Carleton University, Ottawa, Canada. He is now with the Department of Medical Biophysics, University of Toronto, Toronto, Canada (e-mail: matt.hemsley@sri.utoronto.ca).

R. Clackdoyle is with the Univ. Grenoble Alpes, CNRS, Grenoble INP, TIMC-IMAG, 38000 Grenoble, France, and the Department of Physics, Carleton University, Ottawa, Canada. (e-mail: rolf.clackdoyle@univgrenoble-alpes.fr).

E. Ali is with the Department of Physics, Carleton University, Ottawa, Canada, and the Department of Radiology, University of Ottawa, Ottawa Canada (email: elali@toh.ca).

T. Xu is with the Department of Physics, Carleton University, Ottawa, Canada (e-mail: txu@physics.carleton.ca).

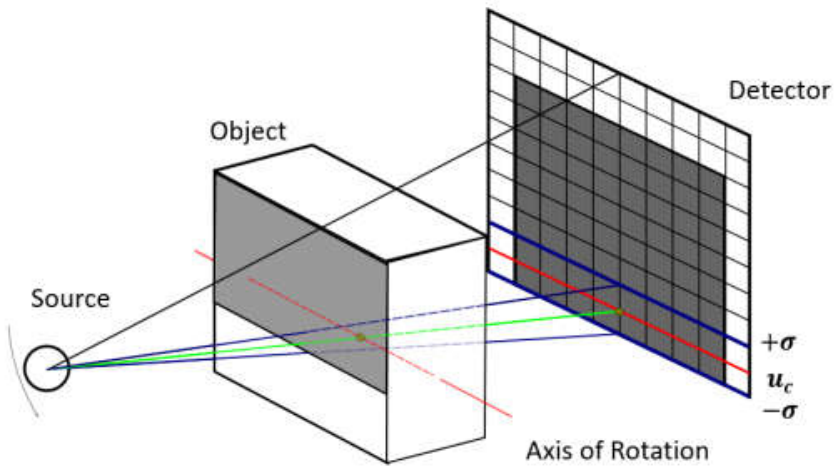

Fig. 1. The offset-detector principle. The x-ray source travels in a circle around the object. The axis of rotation projects to a line on the detector, $u_{c}$. The object is too big to be entirely imaged by the detector. By offsetting, half of the object is seen in one view, whereas the other half of the object is seen in the view taken 180 degrees later in the scan. There is a small overlap region of width $2 \sigma$ centered on $u_{c}$.

projection. Additionally, reconstruction of truncated projections using the widely used Feldkamp-Davis-Kress (FDK) algorithm [2] requires a modification wherein ray sums missing due to truncation are replaced by the closet matching opposite ray sums [3]. This modification relies on robust geometric calibration to be effective. Clinically, a two degrees-of-freedom (2DOF) calibration approach is used in the Elekta XVI CBCT-Linac systems, which measures the shifting of the projection of a point located at the linac isocenter in the two orthogonal directions of the detector at each scanning angle [4]. The benefit of this approach is that it's straightforward to implement in the FDK algorithm by correcting each ray for this shift during backprojection. The drawback is that higher-order misalignment such as variable detector tilting, rotation, and sagging, can't entirely be corrected over the whole field of view with only a 2DOF correction. We present an extended version of an existing phantom-based 9DOF geometric calibration method [5] for CBCT systems capable of accounting for more complex geometric misalignment than the clinical standard 2DOF approach, which we have adapted for offset detector systems and implemented in tandem with the FDK reconstruction algorithm. The method was validated by the successful calibration and reconstruction of a Shepp-Logan phantom with simulated detector misalignments. Previous work by V.G Nguyen [6] also used a phantom-based geometric calibration to determine the projection matrix of the system for use with offset detector positions, however, our method differs in our interpretation and method for determining the geometric 
(a)

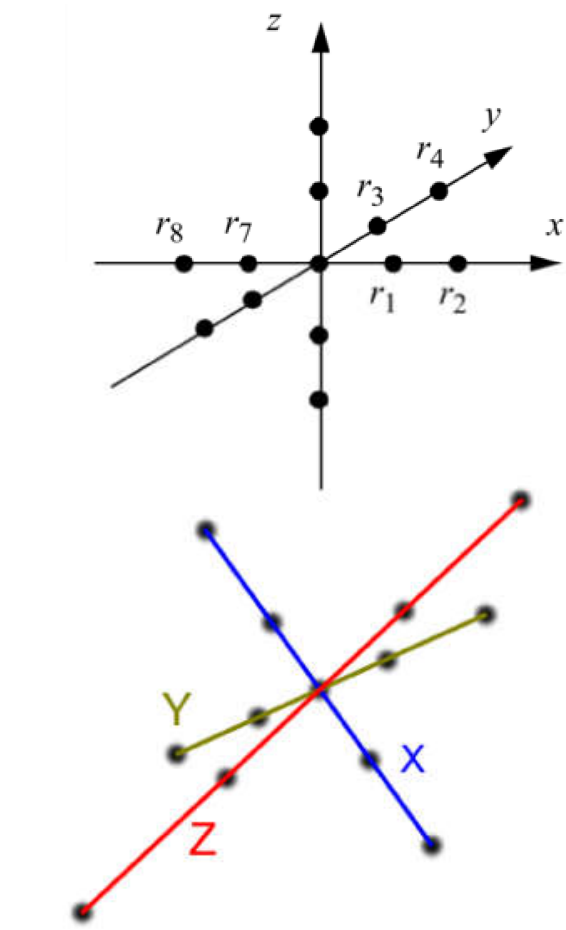

(c)

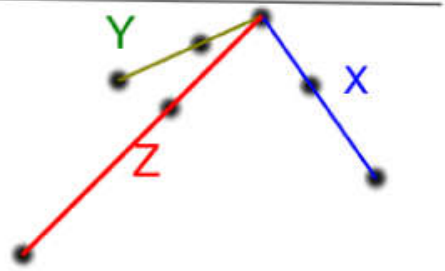

Fig. 2. The 13-ball calibration phantom. (a) In the reference frame of the phantom, the markers are along the axes: $r_{1}=\left(k_{1}, 0,0\right), r_{2}=\left(k_{2}, 0,0\right), r_{3}=$ $\left(k_{3}, 0,0\right), r_{4}=\left(k_{4}, 0,0\right), r_{5}=\left(k_{5}, 0,0\right), r_{6}=\left(k_{6}, 0,0\right)$; and $r_{7}=-r_{1}, r_{8}=$ $-r_{2}$, etc. with $k$ values $\left(k_{1}, k_{2}, k_{3}, k_{4}, k_{5}, k_{6}\right)=(3,7,4,9.5,5.5,13.5)$ all in $\mathrm{cm}$. At the center, $r_{13}=(0,0,0)$. (b) Simulated projection of the phantom onto an artificially large detector. (c) projection of the phantom onto the offset (widthtruncated) detector; part of the phantom projection is truncated.

parameters, a different calibration phantom, and we have shown our method is compatible with the FDK reconstruction, whereas the previous work requires an iterative reconstruction method.

\section{METHODS}

\section{A. Determining Geometric Parameters}

A geometric calibration method proposed by Mennessier et al. [5] and implemented by Spencer et al. [7] was modified for offset detector systems. This calibration method extracts geometric parameters from measured projection images of a specific calibration phantom during a calibration scan to apply to subsequent scans. Our phantom consists of 13 point-like objects at known locations in the reference frame of the phantom as detailed in Fig. 2. As discussed in the following section, our method has three conditions: 1$)$ there must be at least 2 markers on each axis (6 balls total) visible in each projection, 2) each projected marker must be identifiable, even as it enters and exits the FOV due to the offset detector geometry, and 3) the phantom must be placed at isocenter. Condition 1 requires a minimum of 12 small marker balls to be used because nearly half the phantom will project outside the offset detector when the phantom is positioned at isocenter. While not strictly required for the calibration, a $13^{\text {th }}$ ball was included at the origin of the phantom for convenience, so we easily obtain the location of the projection of the isocenter on the detector. In practice, positioning the phantom would be facilitated by the CBCT-Linac laser alignment system (Fig. 9). Condition 2 was achieved through careful choice of the ball-marker locations in the phantom. In our construction, no ball-marker orbits over the 360-degree scan overlap, as seen in Fig. 3, allowing markers to be identified by their location in the projections, provided the (projection-dependent) misalignments are not excessive.

\section{B. Geometric Calibration Phantom}

A brief overview of the original calibration method from [5] is presented here. The parameters which determine the geometry of the systems were extracted from each projection of the calibration phantom. The parameters are, the vector
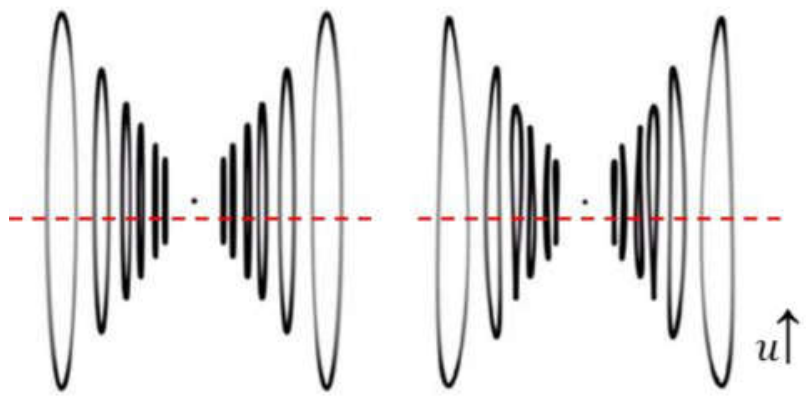

Fig. 3. Left: the trace of calibration phantom marker-ball orbits projected onto an ideal (aligned) detector. Right: the trace of the marker-balls for a misaligned detector. The misaligned detector was shifted by $5 \mathrm{~mm}$ in both directions and a varying in-plane detector rotation was applied. For these traces, ball identification can be automatically achieved using projected orbit locations. Truncation, represented by the dashed lines, occurs perpendicular to the $u$ axis.

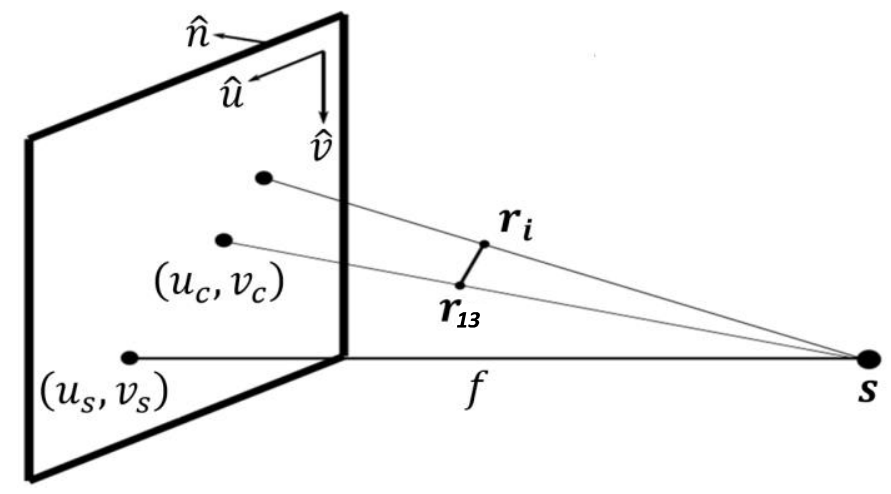

Fig. 4. The geometric parameters of the system to be determined by calibration, as well as a known point $\boldsymbol{r}_{\boldsymbol{i}}$ and phantom center $\boldsymbol{r}_{\mathbf{1 3}}$ are projected onto the detector $\left(u_{c}, v_{c}\right)$. The parameters are distance from the x-ray source to the detector $(f)$; the projection of the source onto the detector $\left(u_{s}, v_{s}\right)$; the three components of the position of the source $(s)$; and the unit vectors which describe the orientation of the detector panel $(\hat{n}, \hat{u}, \hat{v})$, or equivalently the Euler angles describing the detector orientation. 
describing the position of the source, $\boldsymbol{s}$, the projection of the source onto the detector $\left(u_{s}, v_{s}\right)$, the distance from the source to the detector, $f$, and the unit vectors which describe the orientation of the detector $\widehat{\boldsymbol{u}}, \widehat{\boldsymbol{v}}, \widehat{\boldsymbol{n}}$, as shown in Fig. 4. The projection of any point $\boldsymbol{r}$ in the phantom frame onto the detector plane can be found via the mapping

$$
u(\boldsymbol{r})=u_{s}+f \frac{(\boldsymbol{r}-\boldsymbol{s}) \cdot \hat{u}}{(\boldsymbol{r}-\boldsymbol{s}) \cdot \hat{n}} \quad v(\boldsymbol{r})=v_{s}+f \frac{(\boldsymbol{r}-\boldsymbol{s}) \cdot \hat{v}}{(\boldsymbol{r}-\boldsymbol{s}) \cdot \hat{n}}
$$

After translating the detector coordinates so that the isocentre projects to $\left(u_{13}, v_{13}\right)=(0,0)$, (1) can be simplified using intermediate parameters $\boldsymbol{a}=\left(a_{x}, a_{y}, a_{z}\right), \boldsymbol{b}=\left(b_{x}, b_{y} b_{z}\right), \boldsymbol{c}=$ $\left(c_{x}, c_{y}, c_{z}\right)$

$$
u=\frac{a \cdot r}{c \cdot r+1} \quad v=\frac{\boldsymbol{b} \cdot \boldsymbol{r}}{\boldsymbol{c} \cdot \boldsymbol{r}+1}
$$

where $\boldsymbol{a}=\frac{\left(u_{s}-u(\boldsymbol{r})\right) \hat{n}+f \hat{u}}{-\hat{n} \cdot \boldsymbol{s}}, \boldsymbol{b}=\frac{\left(v_{s}-v(\boldsymbol{r})\right) \hat{n}+f \hat{v}}{-\hat{n} \cdot \boldsymbol{s}}, \boldsymbol{c}=\frac{\hat{n}}{-\hat{n} \cdot \boldsymbol{s}}$.

For each projection in the scan of the calibration phantom, we obtain two position values per marker on the detector, $\left(u_{i}, v_{i}\right)$. For the calibration scan, the locations $r_{1}$ to $r_{13}$ were known and the $\left(u_{1}, v_{1}\right)$ to $\left(u_{13}, v_{13}\right)$ were measured, allowing (2) to be solved for vectors $\boldsymbol{a}, \boldsymbol{b}, \boldsymbol{c}$ for each projection. The $\boldsymbol{a}, \boldsymbol{b}, \boldsymbol{c}$ vectors were used for the backprojection step of image reconstruction without conversion back into the original parameters because (2) provides the relationship between the voxel positions $\boldsymbol{r}$ and corresponding detector locations $(u, v)$.

Equation (2) can be readily solved for the vectors $\boldsymbol{a}, \boldsymbol{b}, \boldsymbol{c}$ in the phantom frame because simple linear equations arise due to the structure of the phantom. For example, $u_{1}=$ $a_{x} k_{1}-u_{1} c_{x} k_{1}$ and $v_{1}=b_{x} k_{1}-v_{1} c_{x} k_{1}$ for ball 1 , and similarly for each appearing marker. In our implementation, the $\boldsymbol{a}, \boldsymbol{b}, \boldsymbol{c}$ vectors were obtained from the pseudo-inverse by solving three $2 n \times 3$ linear systems (one for each component of the $\boldsymbol{a}, \boldsymbol{b}, \boldsymbol{c}$ vectors) where $n$ is the number of visible markers present in the projection. The full x-component matrix which occurs when all of the markers on the phantom's $\mathrm{x}$-axis appear is

$$
\left[\begin{array}{l}
u_{1} \\
v_{1} \\
u_{2} \\
v_{2} \\
u_{7} \\
v_{7} \\
u_{8} \\
v_{8}
\end{array}\right]=\left[\begin{array}{ccc}
k_{1} & 0 & -u_{1} k_{1} \\
0 & k_{1} & -v_{1} k_{1} \\
k_{2} & 0 & -u_{2} k_{2} \\
0 & k_{2} & -v_{2} k_{2} \\
k_{7} & 0 & -u_{7} k_{7} \\
0 & k_{7} & -v_{7} k_{7} \\
k_{8} & 0 & -u_{8} k_{8} \\
0 & k_{8} & -v_{8} k_{8}
\end{array}\right]\left[\begin{array}{l}
a_{x} \\
b_{x} \\
c_{x}
\end{array}\right]
$$

The matrices for $y$ and $z$ components are similar. The size of each of these three matrices vary depending on how many markers happen to be in the projection at a given angle, however, as long as the identity of each marker is known and at least two markers on each axis are visible (i.e conditions 1 and 2 are satisfied) then the system will not be underdetermined, and solution yields the required $\boldsymbol{a}, \boldsymbol{b}, \boldsymbol{c}$ vectors.

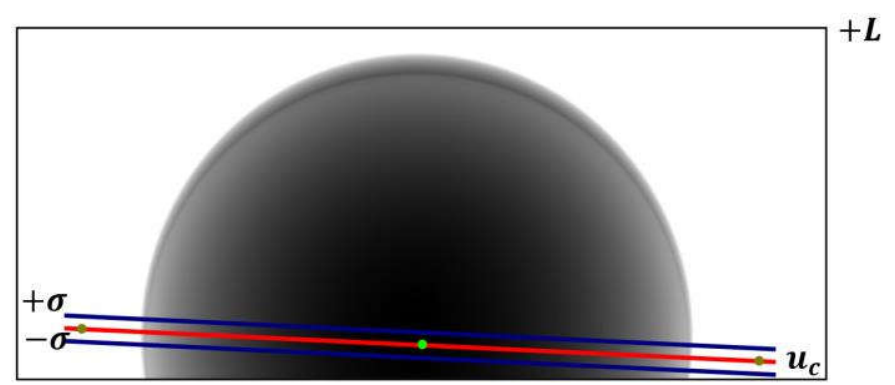

Fig. 5. Truncated projection of a Shepp-Logan phantom overlaid with smoothing weight boundaries from (4). The $u$-direction of (4) is perpendicular to the defined line. The area $u \in(-\sigma, \sigma)$ is the overlap region of the detector, which is in each projection. This method measures $u$ from the projected axis of rotation, which for misaligned detectors is not generally on a line parallel to the edge of the detector. This line, $u_{c}$, is found with (2) and projected onto the detector as shown and used as the origin for the smoothing weight.

\section{Modified Cone-Beam Reconstruction}

We have implemented a variant of the FDK reconstruction algorithm [2], that integrates our geometric calibration to reconstruct from width-truncated (i.e. offset-detector) projections. The geometric calibration aspect of the reconstruction procedure primarily concerns the backprojection step, which we modified to incorporate the calibration information by using the vectors $\boldsymbol{a}, \boldsymbol{b}, \boldsymbol{c}$ and (2). The method for reconstruction of width-truncated projections described by Cho [3] was implemented, where conceptually the missing ray-sums are estimated from the closest matching opposing rays and a smooth weighting scheme was applied for rays in the overlap region near the center. We used the standard scheme, which has the form

$$
w(u)=\left\{\begin{array}{cl}
0 & u \in[-L,-\sigma] \\
1+\sin \left(\frac{\pi u}{2 \sigma}\right) & u \in(-\sigma,+\sigma) \\
2 & u \in[+\sigma,+L]
\end{array}\right.
$$

where, with the offset occurring in the $u$ direction, $u \in$ $(-\sigma, \sigma)$ is the overlap region of the detector and is in each projection, $[-\sigma, L]$ is the area of measured data, and $[-L,-\sigma]$ is discarded by projection truncation but will appear in the opposite projection. This method measures $u$ from the projection of the axis of rotation, assumed known. For misaligned detector panels the projection of the axis of rotation onto the detector is not generally a line parallel to the edge of the detector. To find the true line, (1) is used to determine the location of two additional hypothetical phantom markers, located at points $(1,1,1)$ and $(-1,-1,-1)$ in phantom space. If the center of the phantom is positioned at isocenter, the line defined by these points is the axis of rotation projected onto the detector, as shown in Fig. 5.

\section{Simulations}

To validate our method, simulated projections of the calibration phantom were generated with a non-ideal detector geometry and used to perform the calibration. The determined calibration parameters were then used to reconstruct a SheppLogan phantom that was generated with the same non-ideal geometry. The simulated geometry was modeled after the 


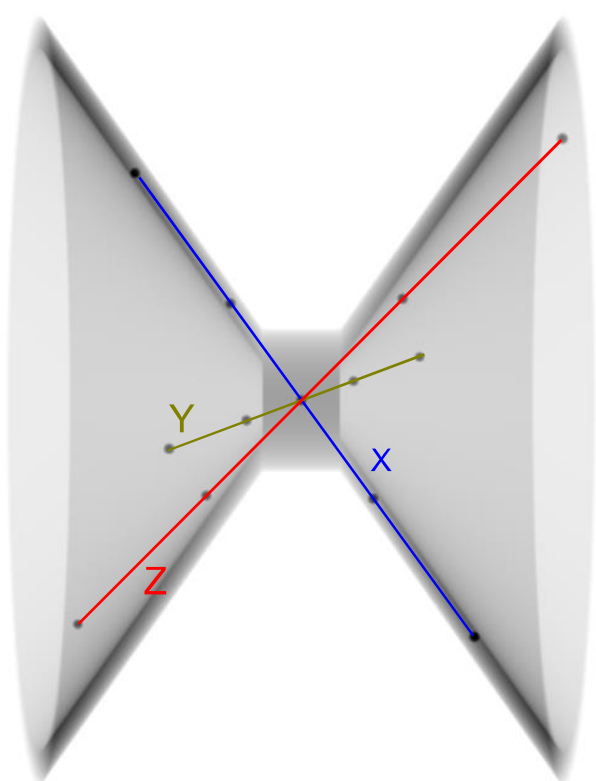

Fig. 6. Non-truncated projection of simulated calibration phantom shown in Fig. 2b), including the plastic shell.

specifications of the Elekta XVI CBCT-Linac [4]. The source to detector distance was $1.536 \mathrm{~m}$, and the source to isocenter distance was $1.000 \mathrm{~m}$. The detector size was 409.6 $\mathrm{mm} \times 409.6 \mathrm{~mm}$ with 1024 isotropic pixels of width $0.4 \mathrm{~mm}$. A total of 348 projections over the 360-degree scan were generated. To create the non-ideal geometry, the center of the detector was shifted in both orthogonal directions of the detector by $5 \mathrm{~mm}$, and the detector was rotated in the detector plan with varying magnitude between $[-1,1]$ degrees over the scan. The overlap region was tested at different values between $\sigma=180$ pixels (minor truncation) to $\sigma=20$ pixels (major truncation).

The calibration was performed by first, extracting the locations of the ball markers projected onto the detector from each projection of the calibration phantom scan. Marker location extraction was done by thresholding the images to eliminate noise and the plastic shell of the phantom (Fig. 6), then using the standard circle detection function in ImageJ [8] to find the center of mass location of each marker. The marker locations for each projection were passed to a Matlab implementation of the calibration [7] to determine the parameters. The parameters were supplied to our modified FDK reconstruction algorithm, written in $C$ and used to reconstruct the non-idealized Shepp-Logan phantom.

A reconstruction was also made of a Shepp-Logan phantom generated with a non-ideal detector, but without applying the calibration as a comparison. In the non-calibrated case, the detector width was doubled (1024x2048 pixels) as the FDK algorithm modified for truncation requires the calibration to define the overlap region. The presented comparison is, therefore, the misaligned and truncated projections corrected with the calibration against an uncorrected misaligned reconstruction, but full-width projections.

\section{RESULTS}

Fig. 7 shows the obtained reconstructions made with a truncation of $\sigma=20$ pixels (i.e with $49.9 \%$ of the phantom truncated in each projection). Without geometric calibration (Fig. 7a, 7b) significant artifacts surrounding the Shepp-Logan objects are observed, whereas the calibrated reconstruction (Fig. 7c, 7d) shows a high degree of spatial fidelity in its ability to resolve the Shepp-Logan objects. In the calibrated reconstructions we do observe the imperfect recovery of the three small ellipsoids and a broad low-level shading artifact (about $0.8 \%$ in intensity) both of which we tentatively (a)

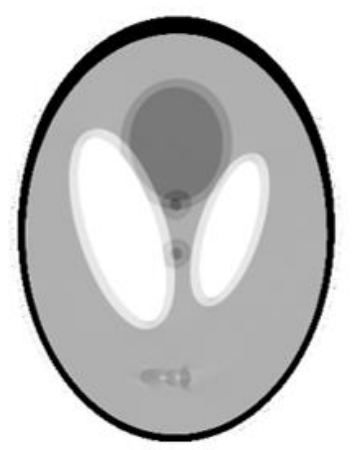

(c)

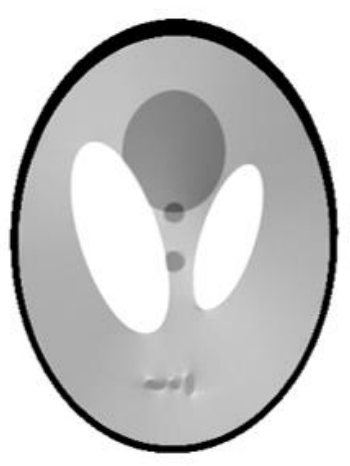

(b)

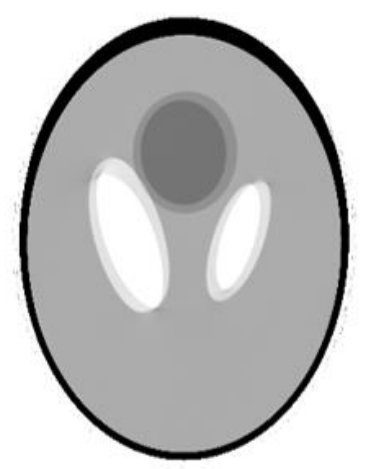

(d)

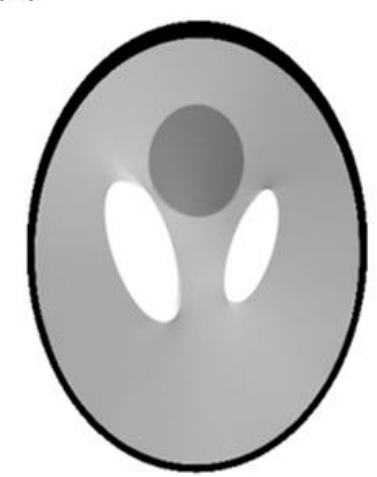

Fig. 7. FDK reconstructions of 3D Shepp-Logan phantom from simulated misaligned projections. (a) and (b) show the reconstruction with uncorrected misalignment only, i.e. no detector truncation (no offset processing needed) while (c) and (d) show corrected misalignment, including offset detector implementation.

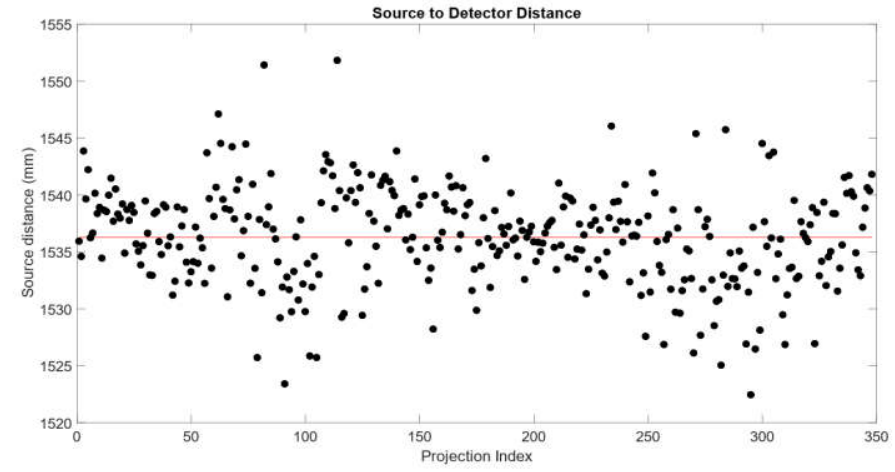

Fig. 8. The source to detector distance determined with the calibration parameters plotted against the projection index of the 348 projections along the 360-degree scan. The mean source to detector distance (red line) determined from the calibration was $(1536+/-4) \mathrm{mm}$. 
attribute to uncalibrated FDK filtering which we have not yet corrected in our current implementation. In addition to the reconstructions, the determined $\boldsymbol{a}, \boldsymbol{b}, \boldsymbol{c}$ calibration vectors were used to recover the specifications of the virtual geometry using (1). Fig. 8 shows the determined source to detector distance as a function of the projection index. The mean source to detector distance over the 348 projections was found to be $(1536+/-4)$ $\mathrm{mm}$, which matches the true value of $1536 \mathrm{~mm}$.

\section{DISCUSSION}

We have presented a phantom-based method CBCT geometric calibration which is applicable to offset detector panels, as well as compatible with the widely used FDK reconstruction algorithm. We reconstructed a simulated 3D Shepp-Logan phantom generated with a misaligned scanner geometry involving shifting and variable rotation of the detector panel. Our method applies to other types of detector misalignment, such as source to detector distance, however, we do assume that the x-ray source travels along a perfect circle. Our results of the calibrated and truncated Shepp-Logan phantom versus the uncalibrated but full-width phantom show that despite being truncated, the Shepp-Logan objects are more clearly recovered and geometrically accurate in the calibrated reconstruction. An attempt to approximate the line of rotation using a 2DOF approach was shown to lead to significant artifacts at high amounts of truncation, which disqualified this approach from inclusion in the comparison. This implies that a calibration method with more than 2DOF could be beneficial for reconstrcuting projections with extreme truncation (approaching $\sigma=0$ ) as well as for severly misaligned detectors. The calibration was also validated by the recovery of the true geometric parameters the system was simulated with. Noise in Fig. 8 is hypothesised to be introduced when the calibration marker locations were extracted from the projections.

Positioning the phantom at isocenter (condition 3) is not strictly required for the calibration, but in combination with the inclusion of the $r_{13}$ marker such positioning allows for easy determination of the projection of the isocenter onto the detector, $\left(u_{c}, v_{c}\right)$. This condition could be alleviated by using the known marker locations in phantom space and their projected locations on the detector to determine $\left(u_{c}, v_{c}\right)$ analytically, similar to [5] and [7]. However, the phantom was designed to satisfy the additional conditions 1 and 2 when placed at isocenter, so while an analytical method to determine $\left(u_{c}, v_{c}\right)$ would alleviate the need for condition 3 , the positioning of the phantom is still somewhat limited.

This work is of clinical importance in the context of imageguided radiation therapy due to the need for accurate reconstructions from projections acquired with offset detector commonly obtained with CBCT-Linacs.

We have constructed a physical version of the calibration phantom, to validate the method with real data (Fig. 9).

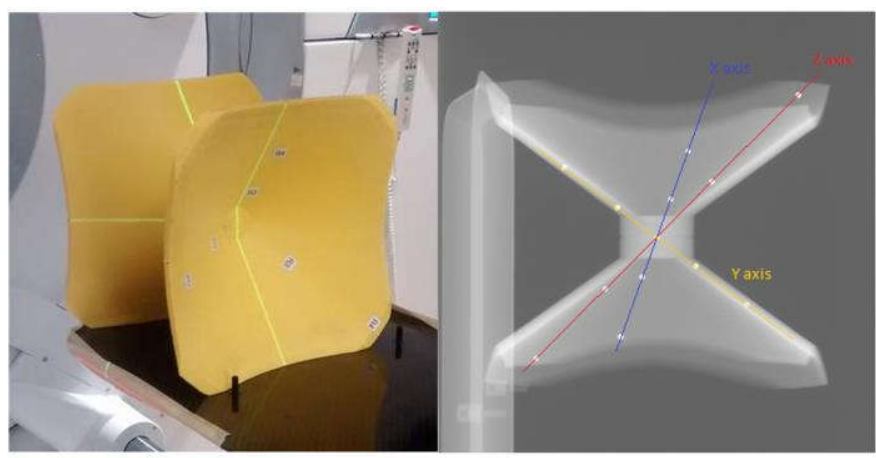

Fig. 9. Physical calibration phantom. The plastic shell was 3D printed as a single piece. Tungsten-carbide ball markers were implanted with a precision of $0.05 \mathrm{~mm}$.

\section{REFERENCES}

[1] D.Verellen, M.D.Ridder, K.Tournel, M.Duchateau, T.Reynders, T.Gevaert, N.Linthout, and G.Storme, "An overview of volumetric imaging technologies and their quality assurance for IGRT," Acta Oncol, vol. 47, no. 7, pp. 1271-1278, 2008

[2] L.A.Feldkamp, L.C.Davis, and J.W.Kress, "Practical cone-beam algorithm," J Opt Soc Am A, vol. 1, no. 6, pp. 612-9, 1984.

[3] P.S.Cho, R.H.Johnson, and T.W.Griffin, "Cone-beam CT for radiotherapy applications," Phys Med Biol, vol. 40, no. 11, pp. 1863-83, 1995.

[4] XVI R4.5 Corrective Maintenance Manual. ELEKTA, 2010.

[5] C.Mennessier, R.Clackdoyle, and F.Noo, "Direct determination of geometric alignment parameters for cone-beam scanners," Phys Med Biol, vol. 54, no. 6, pp. 1633-60, 2009.

[6] V.-G.Nguyen, "View-dependent geometric calibration for offset flatpanel cone beam computed tomography systems," Opt Eng, vol. 55, no. 4, 043102, 2016

[7] B.Spencer, R.Clackdoyle, C.Mennessier, and T.Xu, "Distortion correction, geometric calibration, and volume reconstruction for an isocentric C-arm x-ray system: Preliminary studies," Proc 2nd international conference on image formation in X-ray computed tomography. Salt Lake City UT, June 2012. pp. 279-283.

[8] C.A. Schneider, W.S. Rasband, and K.W. Eliceiri, "NIH Image to ImageJ: 25 years of image analysis". Nature Methods 9, pp. 671-675, 2012. 